\title{
Propellant Mass Gauging via Modal Analysis on the International Space Station
}

\author{
Taylor Peterson \\ Carthage College \\ Internship Final Report ${ }^{1}$ \\ NASA Kennedy Space Center \\ NE-L6 Advanced Engineering Development Branch \\ Mentor: Dr. Edwin Cortés \\ Summer 2020
}

\begin{abstract}
In order for NASA to obtain sustained human presence in space, a higher fuel gauging accuracy is required. Current gauging methods have a sub 5-10\% error which requires an additional fuel margin for any rocket or satellite. Doing this increases weight, therefore creating a higher cost. Gauging technologies capable of solving this problem either do not exist or only exist at low Technology Readiness Levels (TRLs). The Modal Propellant Gauging (MPG) project is an effort to develop a higher resolution, light-weight, and cost efficient fuel gauging method for lowgravity environments [2]. MPG uses excited surface acoustic waves on the outside of the tank to measure the tanks resonant frequencies. In microgravity, the liquid will adhere to and coat the inner walls of the tank. The resonant mode frequencies are sensitive to the presence of liquid, meaning the accuracy of MPG is amplified in low-gravity. Modal measurements are taken using sensors adhered to the outer tank walls and a small data acquisition system. A Frequency Response Function (FRF) is then generated to create a reading for a fuel gauge.

The newest iteration of MPG is Modal Propellant Gauging - International Space Station (MPG-ISS), which will undergo 30 days of autonomous testing onboard the International Space Station (ISS). This payload will implement MPG onto two tanks separated into two ISS payload lockers. A set amount of liquid will be transferred between both tanks as data is taken on both and stored on the in-flight computer. The MPG-ISS experiment will help validate the use of MPG on future space mission, such as Artemis. This experiment is the next step for MPG to be used on future mission to the Moon and Mars.
\end{abstract}

\section{Introduction}

For over the past fifty years, propellant mass gauging has been a notorious problem in spacecraft. During the Apollo 11 Moon landing, the mission was almost a disaster due to an inaccurate fuel gauge reading. To remedy this problem, Carthage College, NASA Kennedy and NASA Johnson have been working together to develop the MPG technology. MPG is a lightweight and non-invasive method of measure propellant in microgravity. Through student led efforts, MPG has undergone various parabolic flight campaigns with the Zero Gravity Corporation and a handful of suborbital flights with Blue Origin. As the data continues to come back promising, NASA requires a longer duration of autonomous testing in microgravity. The MPG-ISS experiment will be onboard the ISS for 30 continuous days and run a series of unmanned tests to prove the reliability of the MPG technology.

My summer internship at NASA Kennedy Space Center focused on designing and 3D modeling the MPG-ISS experiment. MPG is being considered for use on the Orion Spacecraft, the Lunar Gateway Power and Propulsion Element (PPE), and the Gateway ascent modules. MPG has seen many iterations for testing, however this experiment will give the necessary data to show the accuracy of MPG in long-term use. Since this is an objective that no other student-led MPG project has touched, the design phase has been an extreme challenge, adding in new and improved components such as the data acquisition system and the electrical/communication interface, described in a later section.

\footnotetext{
${ }^{1}$ Funding provided by the WSGC under Center Internship KSC NE-L6
} 
Purpose MPG is an effort to introduce a highly accurate and cost effective method of measuring propellant in low gravity. A previous iteration of this technology has seen multiple manned parabolic flights, allowing for 22 seconds of low gravity at a time. Through this, MPG has proven to work and been improved over the last decade. In result of these flights, NASA showed great interest the duration it takes for the liquid to reach equilibrium. To test this, another iteration of MPG was manifested to fly twice on Blue Origin's New Shepard Vehicle in 2019, which allowed for about 3 minutes in low gravity conditions.

In order for MPG to be considered flight-ready for use on the Gateway PPE, extensive testing in microgravity must be preformed with a constant flow of liquid. The objective of MPG-ISS is (1) to identify the variety and occurrence frequency of equilibrium liquid surface configurations with the modeled propellant tank geometries in microgravity, (2) to show that the liquid can equilibrate in different configurations at the same fill fraction while MPG associates the correct fill level to each configuration and (3) to evaluate the performance of three separate gauging algorithms developed for MPG and determine their validity for settled and sloshing liquid, static and dynamic fill fractions in low gravity.

Concept The MPG technology uses acoustic surface waves to determine the amount of liquid inside propellant tanks. In microgravity, liquid will adhere to the inner walls due to high surface tension and "mass-load" the tank walls. This causes the tank and liquid to act as the same system with one mass and allow for the MPG technology to accurately gauge the amount of liquid within a 1-5\% error, and has even seen an average accuracy as low as $0.24 \%$ [3].

MPG uses Experimental Modal Analysis (EMA) techniques to correlate the modal response of a 1- $g$ surface to the surface response at the same fluid fill fraction in microgravity environments. Every object has a set of natural resonance frequencies determined by its mass, material, and geometry. As the fuel in the tank depletes, the responsive frequencies gets higher. Due to an increase in electrical noise at higher frequencies, the data analysis focuses on 500-1500 $\mathrm{Hz}$ [4]. EMA involves subjecting the exterior tank wall to a broad-band white noise signal with a range of 50-3,000 Hz. This signal is delivered via a PZT actuator, labeled in Figure 1, that excites all resonant frequencies and modes of the tank. A PZT actuator is a flexible patch that converts the electrical signal to a mechanical energy, or vibration. The signal is amplified by piezo driver modules to an RMS amplitude of 100V [1]. Situated right next to the actuator is a PZT sensor that acts as a "monitor", recording the amplified white noise signal from the actuator. On the opposite side of the tank is a second PZT sensor labeled as the "sensor". The sensor records the weakened white noise signal and the amplified resonant response frequency of the tank. The Fast Fourier Transform (FFT) is taken of the monitor and sensor input to generate FRFs.

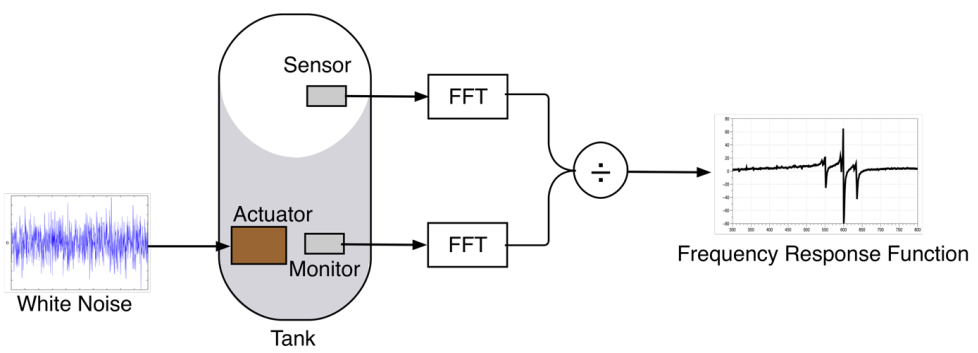

Figure 1: Experimental concept. A white noise signal is sent to a PZT actuator and recorded by the PZT "monitor". The PZT "sensor" records the amplified resonant frequencies and modes of the tank. The FFT of the monitor and sensor is taken to generate an FRF to determine the liquid fill fraction inside the tank.

\section{Payload Materials and Design}

The MPG-ISS experiment consists of two modeled propellant tanks that will have a flow loop to exchange liquid between them, a data acquisition system, control hardware, and two lockers that will contain the entire payload. The experiment has many crucial design constraints given by the EXpedite the PRocessing of Experiments to the Space Station (EXPRESS) program. This includes limits on weight, hardware and liquid volume, heat, power, etc. The 
inside of one single EXPRESS locker is $2 \mathrm{ft}^{2}$ [5].

ISS EXPRESS Rack Since this experiment will be on-board the ISS for an extended period of time, it will be contained in two payload lockers. NASA follows the modular EXPRESS rack system that allows for experiments to be implemented on the ISS using EXPRESS lockers. There are multiple racks of eight lockers with various connections from the lockers to the EXPRESS racks [5]. The lockers come in single or double size and MPG-ISS will consist of two single size lockers, one is pictured in Figure 2. They are aluminum lockers with three removable panels on the front to allow for customizable layouts for various connectors, switches and LED lights. Having the experiment across two single EXPRESS lockers will allow for larger modeled propellant tanks and more realistic flow/equilibrium dynamics. Since there are two lockers in this experiment and they will have different components, they are labeled Locker A and Locker B.

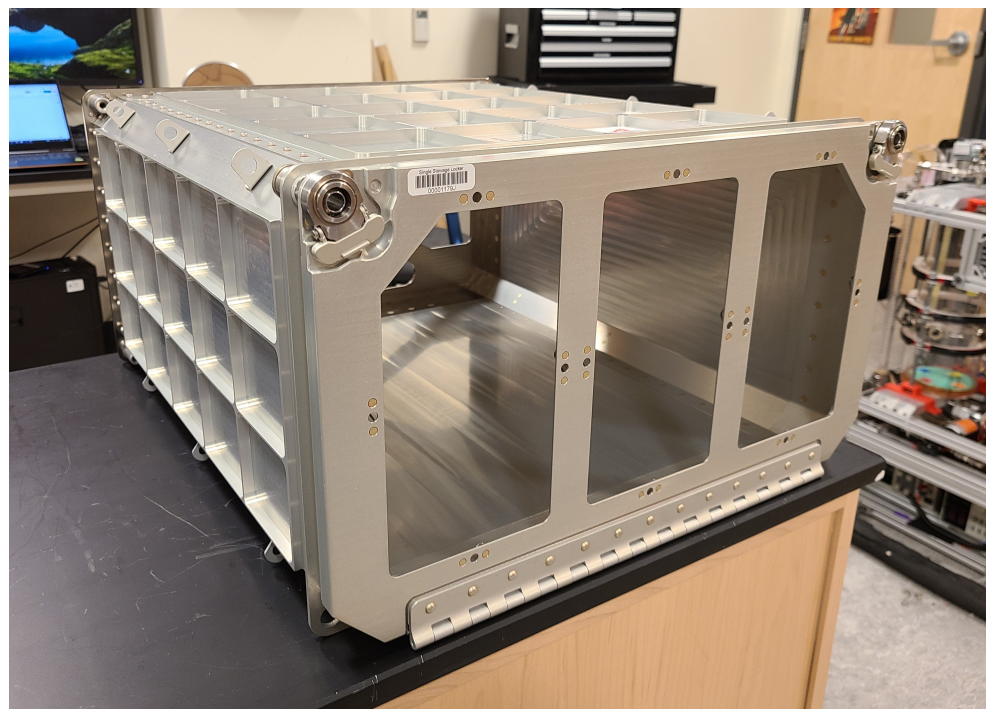

Figure 2: Empty single size EXPRESS locker.

Flow Loop The MPG-ISS experiment will simulate propellant refueling in microgravity by flowing liquid between two tanks. This will be done by utilizing a flow loop constructed with a few pumps, solenoid valves, and fittings to allow for a steady exchange of liquid between the tanks, as shown in Figure 3. There are four manual valves shown, two for filling and draining liquid from the experiment and the other two are for pressure release in the system. These valves are for ground use only and will not be opened during flight. Since the final flow loop will be closed, there will be no need to equalize pressure. On the end of each tank will be a pressure transducer to record the pressure data during ground testing and in-flight operations.

Part of my internship was dedicated to sourcing and testing the correct parts for the flow loop to ensure the least amount of adaptors to ensure steady flow, prevent leaking, and cut down on weight. Both lockers will have a similar set up for the flow loop. Due to constraints from NASA and the EXPRESS program, the total volume across both tanks will be just under one gallon of water. All components of the flow loops will be pressure tested before flight and there will be two on-board pressure transducers to record pressure data for ground testing and in-flight operations. As afore mentioned, the experiment will hold two modeled propellant tanks. As there is a labeled Locker A and Locker B, the tanks are labeled Tank A and Tank B respectively, shown in Figure 3. Tank B will have a slightly smaller volume than Tank A to ensure that Tank B will go from a 100\% fill fraction to a nearly $0 \%$ fill for extensive data analysis. 


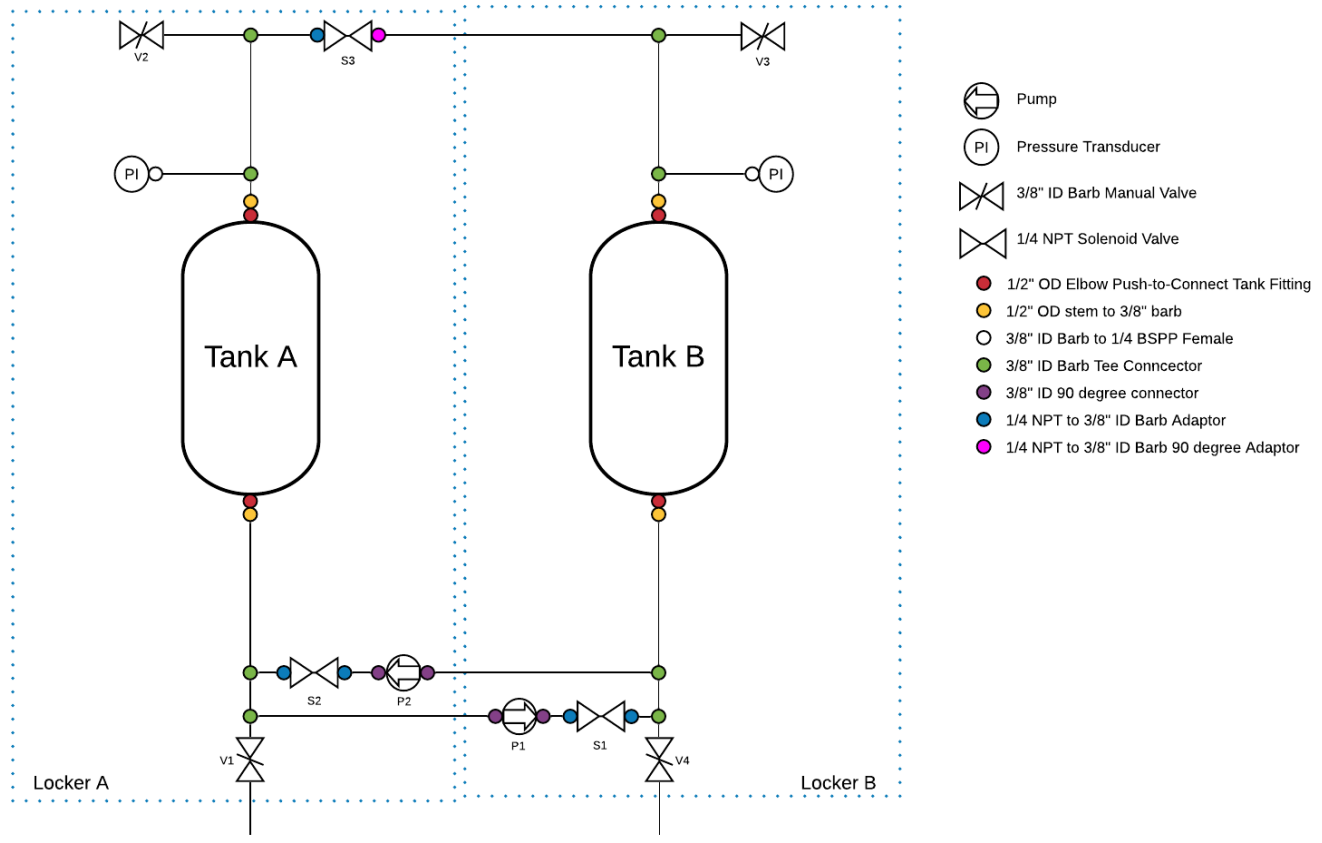

Figure 3: Connector chart in Locker A and B for the MPG-ISS flow loop.

Propellant Management Device Both simulated propellant tanks used in MPG-ISS will contain a vane and ring Propellant Management Device (PMD), shown in Figure 4. The purpose of the PMD is to attempt to control the location of the liquid in low gravity environments. The PMD will be attached to the drain port of each tank in attempt to navigate the liquid to the port. Once the tank reaches a low fill fraction, the vane and sponge design of the PMD will ensure separation of liquid and gas near the outlet. This will also assist in reaching lower fill fractions for data analysis. There will be two onboard GoPro cameras (one in each locker) to record video data the entire flight, and two Raspberry Pi cameras (one in each locker) providing live data to ground for observing the tank liquid level and configuration.

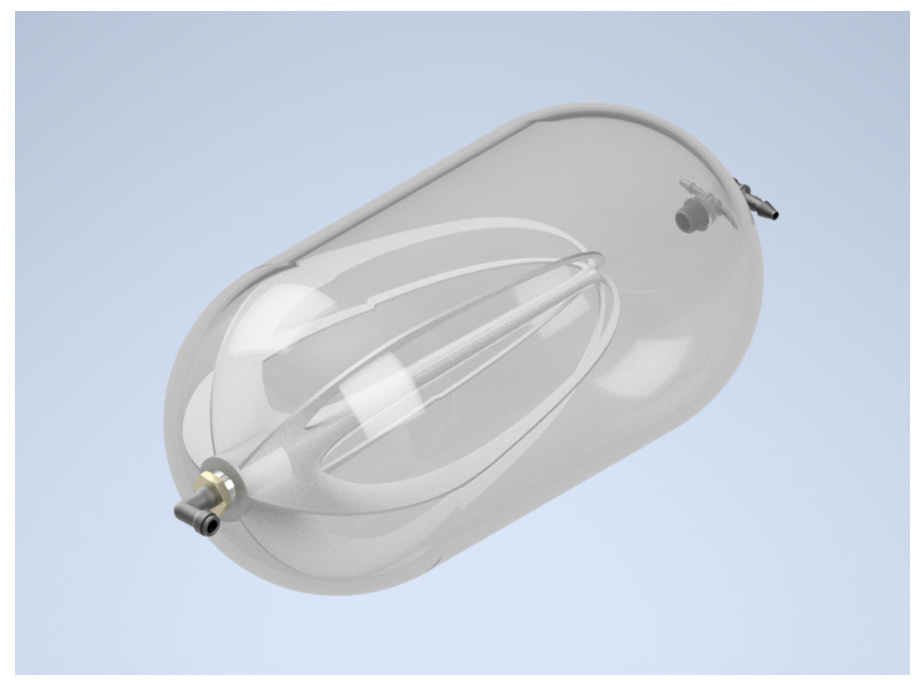

Figure 4: Tank A with a Propellant Management Device connected to the drain port. 
Tank Mounts An important aspect that was considered during the design phase for this project was mounting the tanks. A critical constraint given by the nature of the experiment is limiting the amount of pressure on the body of the tank, as it can change the frequency response output. To avoid this, the tank is held by the bulkhead on either end of the tank using a custom built bracket made of aluminum, shown in Figure 5. This brackets has bends on either ends so additional corner brackets, mounted to the wall, can hold it in place. Both sets of brackets have a long slit to tighten the tank into place for flight. Not only does this design allow for minimal pressure on the tank, but easy installation and removal of the tank. The same design is used in both Locker A and Locker B.

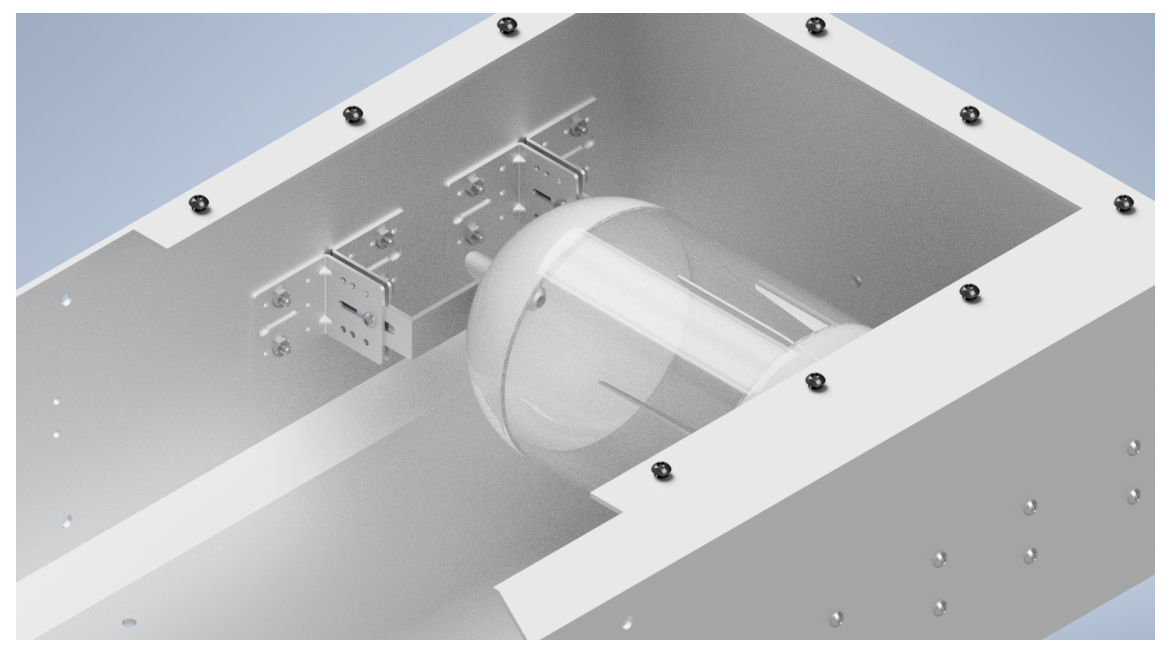

Figure 5: The tank is mounted with various corner brackets and custom brackets fitting around the tank bulkheads, allowing for no additional pressure to be added on the tank walls.

Front Panel Since the MPG-ISS experiment will have two lockers, they are required to communicate both with each other and with the ISS EXPRESS rack via the front panel connections, shown in Figure 6. To connect to the rack, there will be a main power connection and an ethernet port on Locker A. From here, Locker A will also have power and data connections to Locker B to supply the second locker with power and for data storage/retrieval. Both lockers will have four fluid connections for the liquid to fill and drain between the two tanks. To prevent leaking, panel mounting quick disconnects are used on either side of the front panels.

Along with connections, the front panel will also hold various switches and LED lights. First, there is a power switch to turn on power to the entire payload once the power line is plugged in. To start any testing, there is a 'Start' switch to tell the onboard computer to begin the testing cycle. If there is ever an issue with testing or any components, there is a 'Restart' switch that will cycle power to the entire payload. All of the connections and switches are labeled accordingly for ease of use when the astronauts onboard the ISS initially set up the experiment. Any switches shown in Figure 6 will have protective guards so none are triggered on accident while onboard the ISS. To save space and create easier wiring, the front panel connections are mounted on the front of the electronics box. 


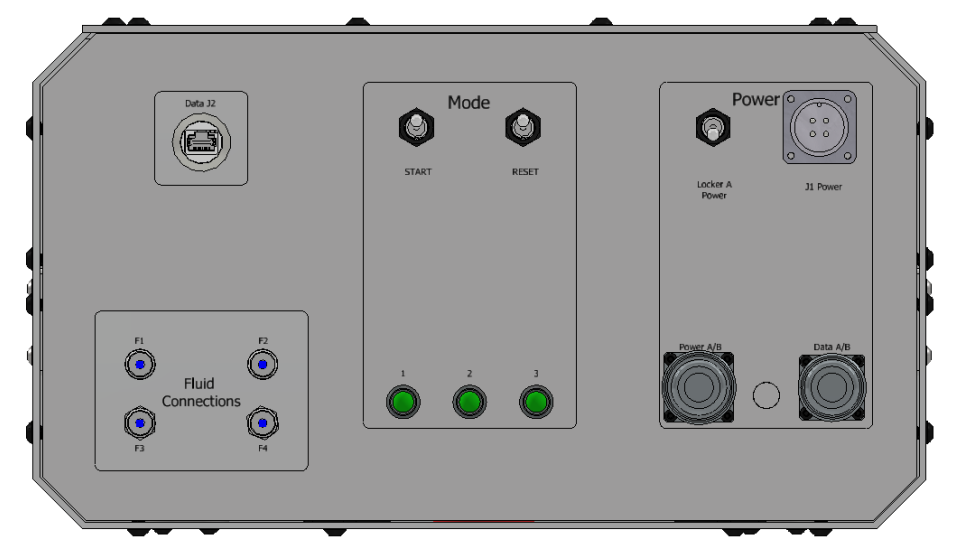

Figure 6: The front panel connections on Locker A showing the various connections, switches, and LED lights.

Electronics The electronics are a critical part of the payload. Most of the components will be kept inside of the electronics box which is kept near the front of the ISS locker. Figure 7 shown the components mounted inside the electronics box on two aluminum panels. The parts consist of voltage converters, a Raspberry Pi as the onboard computer and white noise generator, a noise amplifier, a PWM, relay modules, and an EMI filter. Several pieces have custom made parts for easier mounting together and to allow a small fan and heat sink for thermal issues. Some of these parts connect to components outside of the electronics box, such as the GoPro cameras, the flow loop parts, and the tank sensors.

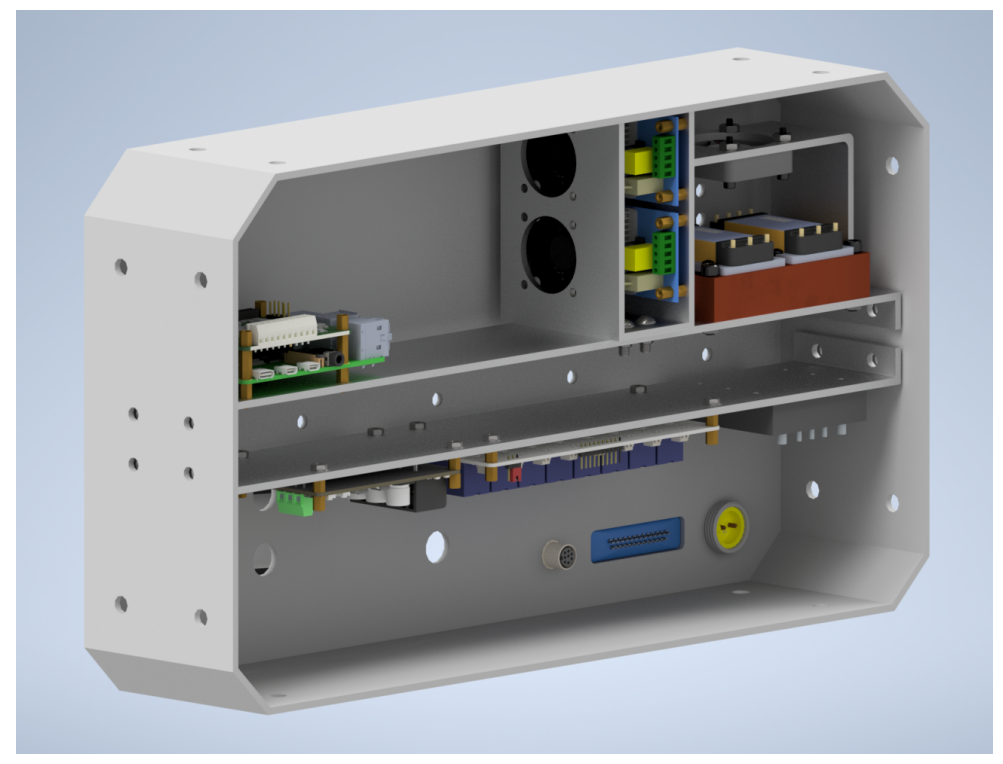

Figure 7: A view showing the various components mounted inside the electronics box.

Full Locker All of the sections talked about above all come together inside of the aluminum locker walls to contain the experiment. The electronics box is held in the front for front panel connections and sealing the front. The tank is in the back of the locker to allow the GoPro camera to capture footage of the entire thing. The flow loop components are fits inside the locker as needed to not be in the way of the cameras or any connections. This design has gone through many iterations and constantly being improved. Once in the final version, it will demonstrate the MPG technology on the ISS. 


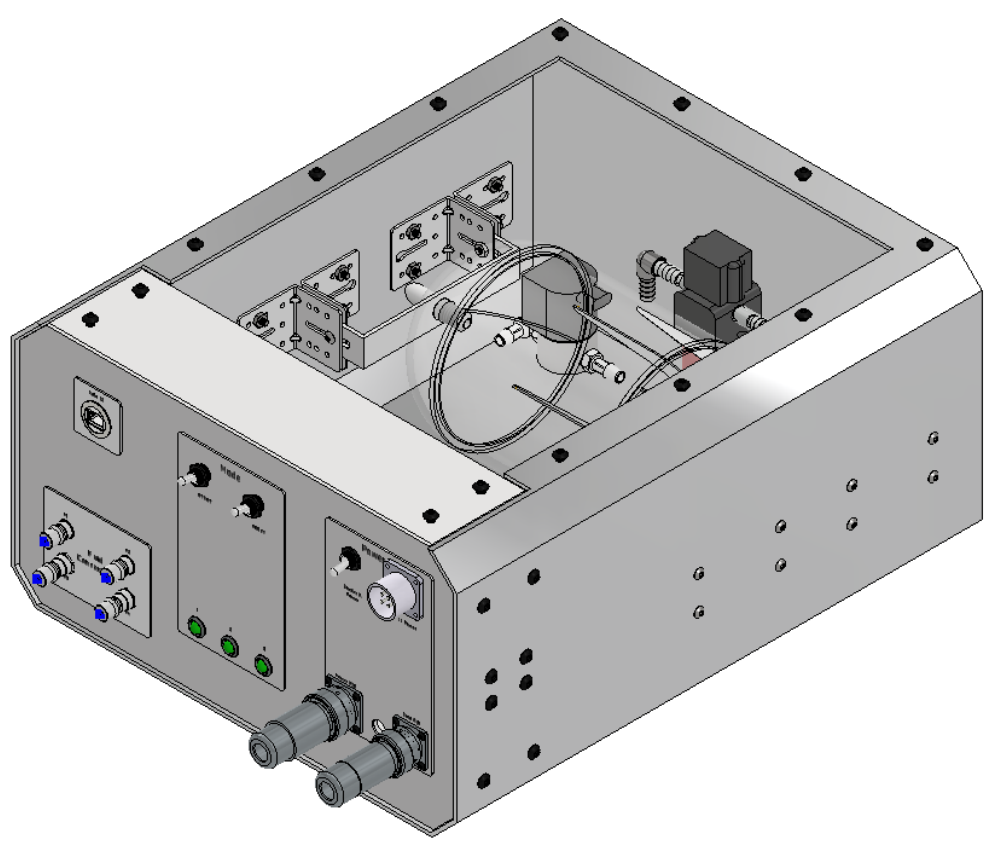

Figure 8: A 3D CAD view of Locker A in its entirety, without the top panel.

\section{Conclusion}

MPG is a lightweight and cost efficient solution to current gauging inaccuracies. My summer internship at NASA KSC consisted of assisting in the development of the MPG-ISS payload, a continuation of the existing MPG experiments. Through this internship, I gained a great deal of knowledge of 3D CAD modeling in Autodesk Inventor along with essential time management skills. NASA KSC provided me with an excellent virtual internship experience, while allowing me to design and create an experiment that will help further NASA's mission to the Moon and Mars.

The MPG-ISS experiment is an essential part of NASA's mission of furthering human space exploration. In order for sustained human presence in space, a higher gauging accuracy is required. MPG has already been proved to work in up to 3 minutes of microgravity. NASA is now interested in a longer microgravity duration, and the MPG-ISS experiment will answer any questions open about the reliability of MPG. This is a critical experiment and will assist NASA in being one step closer to putting humankind back on the Moon, and eventually Mars.

\section{References}

[1] Kevin M. Crosby et al. "Modal Propellant Gauging: High-resolution and non-invasive gauging of both settled and unsettled liquids in reduced gravity". In: Europe PMC (2019).

[2] Kevin Crosby and Rudy Werlink. "“'Experiment Requirements Document: Modal Propellant Gauging (MPGISS): Establishing the Fill Resolution of MPG During Continuous Drain Operation in Microgravity”. 2020.

[3] Kevin Crosby, Rudy Werlink, and Eric Hurlbert. "Liquid Propellant Mass Measurement in Microgravity". In: Gravitational and Space Research (2021).

[4] Taylor Peterson, Cassandra Bossong, and Bennett Bartel. "Modal Propellant Gauging in Microgravity". In: Proceedings of the Wisconsin Space Conference (2019).

[5] W. S. Thompson and Lake E. R. "Conducting Research on the International Space Station using the EXPRESS Rack Facilities”. In: 30th American Society for Gravitational and Space Research (2014). 